Original Article

\title{
Self-reported Exhaustion is Associated with Small Life Space in Older Adults with Mild Cognitive Impairment
}

\author{
Kota Tsutsumimoto, MSc, $\mathrm{PT}^{1,2)^{*}}$, TAkehiko Doi, MSc, PhD, $\mathrm{PT}^{2)}$, \\ Hiroyuki Shimada, MSc, PhD, $\mathrm{PT}^{2)}$, Hyuma Makizako, MSc, PgD, $\mathrm{PT}^{2)}$, \\ Kazuki Uemura, MSc, PhD, $\mathrm{PT}^{2}$, Hiroshi Ando, MD ${ }^{1)}$, Takao Suzuki, MD ${ }^{3)}$ \\ 1) Department of Rehabilitation Science, Kobe University Graduate School of Health Sciences: 7-10-2 \\ Tomogaoka, Suma-ku, Kobe, Hyogo 654-0142, Japan \\ 2) Department of Functioning Activation, Center for Gerontology and Social Science, National Center \\ for Geriatrics and Gerontology, Japan \\ 3) Research Institute, National Center for Geriatrics and Gerontology, Japan
}

\begin{abstract}
Purpose] Older adults experience exhaustion-induced health problems, such as poor physical function and low physical activity levels. The associations between self-reported exhaustion and physical function and activity are not clear in older adults with mild cognitive impairment (MCI). The aim of this study was to investigate the relationships between self-reported exhaustion and physical function and activity in older adults with mild cognitive impairment. [Subjects] A total of 356 older adults with mild cognitive impairment (mean age $=71.6 \pm 0.3$ years, $50.8 \%$ women) were included in this study. [Methods] Self-reported exhaustion was identified by one item from the Study of Osteoporotic Fractures index. Gait speed, gait endurance, and life space were also assessed. [Results] Sixty-two participants reported having exhaustion, giving a $17.4 \%$ prevalence of self-reported exhaustion among these individuals. Logistic regression analysis showed that the Life-Space Assessment score was the only parameter significantly independently associated with exhaustion status (adjusted odds ratio $0.97,95 \%$ confidence interval 0.95-0.99). [Conclusion] These results suggest that self-reported exhaustion is associated with life space. Future research is needed to identify ways for older people with MCI to improve their exhaustion status.

Key words: Mild cognitive impairment, Self-reported exhaustion, Life space
\end{abstract}

(This article was submitted May 20, 2014, and was accepted Jun. 23, 2014)

\section{INTRODUCTION}

Mild cognitive impairment (MCI) is considered to be the transitional phase between normal aging and dementia and is recognized as the prodromal stage of Alzheimer's disease (AD), which is the most common form of dementia ${ }^{1)}$. Evidence from neuropsychological and neuroimaging studies have suggested that mild cognitive impairment (MCI) represents a clinical prodromal status for degenerative dementias such as $\mathrm{AD}^{2}$. For example, a population-based study in Sweden reported that the relative risks of progression to dementia in a 3 year follow-up in subjects with mild, moderate, and severe cognitive impairment without dementia were $3.6,5.4$, and 7.0 , respectively ${ }^{3}$. Older people with $\mathrm{MCI}$ show not only cognitive decline but also frailty, which is one of the most crucial health issues among older adults ${ }^{4)}$.

The concept of frailty comprises five general factors:

*Corresponding author. Kota Tsutsumimoto (E-mail: k-tsutsu@ncgg.go.jp)

C2014 The Society of Physical Therapy Science. Published by IPEC Inc. This is an open-access article distributed under the terms of the Creative Commons Attribution Non-Commercial No Derivatives (by-ncnd) License $<$ http://creativecommons.org/licenses/by-nc-nd/3.0/> shrinking, weakness, exhaustion, slowness, and a low activity level ${ }^{5,6}$. Older adults with cognitive impairment, including MCI, are at higher risk of becoming frail than older adults who are cognitive healthy. In addition, older non-frail individuals with lower cognitive scores are significantly more likely to acquire one or more components of frailty over 10 years compared with those with higher cognitive scores $^{7)}$. In turn, older adults with cognitive decline, such as MCI, are more vulnerable, so it is not enough to approach them from the standpoint of cognitive function only.

Among the five factors of the frailty concept, exhaustion in particular contributes to a more rapid onset of frailty and affects $80 \%$ of frail older adults ${ }^{8)}$. Based on self-reported symptoms, exhaustion was defined as a condition characterized by unusual fatigue or a general loss of energy. Exhaustion in the older population results from lower physiological capacity, as reflected in less total energy expenditure and a low physiological reserve compared with younger people ${ }^{9}$. These effects lead to an inactive lifestyle that is accompanied by behavioral changes to save energy, thus preventing exhaustion from daily activities ${ }^{9}$, and a subsequent decline in physical or cognitive function. In fact, self-reported exhaustion negatively affects functions among older people: ${\text { gait } \text { speed }^{10)} \text {, gait endurance }}^{11)}$, physical activity ${ }^{12)}$, and 
cognitive function ${ }^{13)}$. So, self-reported exhaustion in older people is one of the health problems to prevent.

In older adults with MCI, maintaining physical performance through a physically active lifestyle may play an important role in delaying or preventing dementia ${ }^{14}$. It remains unclear whether or not older people with MCI have the association with self-reported exhaustion, although selfreported exhaustion was found to be negatively associated with physical function and physical activity among healthy older adults. Thus, it is important to clarify the relationship between exhaustion and physical function, including physical activity, in older adults with MCI. The first aim of this study was to investigate the prevalence of exhaustion among older adults with MCI and to compare it with the prevalence of exhaustion in the elderly population, which is estimated to be about $20 \%{ }^{15}$. The second aim of this study was to investigate the prevalence of self-reported exhaustion among older adults with MCI and whether exhaustion is related to physical ability and physical activity levels.

\section{SUBJECTS AND METHODS}

The study population and data in this study were obtained at baseline in a larger cohort study, the Obu Study of Health Promotion for the Elderly (OSHPE) ${ }^{16)}$. The classification of an individual as having MCI was based on the consensus previously published by Petersen ${ }^{1)}$. These criteria included (1) a subjective memory complaint, (2) objective cognitive decline, (3) intact general cognitive function (mini-mental state examination (MMSE) score $>23)^{17)}$, (4) ability to perform activities of daily living (ADL) independently, and (5) absence of clinical criteria indicating dementia. Objective cognitive decline was defined as being indicated by a score of more than 1.5 standard deviations from the score for healthy in the OSHPE database after adjustment for age ${ }^{16)}$. We assessed objective cognitive function using the National Center for Geriatrics and Gerontology Functional Assessment Tool. This software is used on a tablet PC and contains a battery of cognitive tests; the details of these tests have been described in detail elsewhere ${ }^{18)}$. The tests comprise eight tasks to assess memory, attention, executive memory, processing speed, and visuospatial skills. Six hundred forty-nine older adults with MCI were selected from the larger cohort study as the potential study population for this study. After applying the exclusion criteria, 356 people with MCI were deemed suitable to participate in the current study. The exclusion criteria were: (1) history of cerebrovascular disease, Parkinson's disease, depression, or connective tissue disease; (2) presence of a heart pacemaker; (3) severe auditory or visual deficit; (4) depression symptoms (Geriatric Depression Scale-15 score $>5)^{19)}$; or (5) prior commitment to other studies. Informed consent was obtained from all participants before inclusion in the study, and the Ethics Committee of the National Center for Gerontology and Geriatrics approved the study protocol.

Demographic data were collected including age, sex, number of medications, and body mass index (BMI). Selfreported exhaustion was identified using a questionnaire "Do you feel full of energy?" from the Study of Osteopo- rotic Fractures (SOF) index ${ }^{5)}$. Participants who responded "No" to the question were placed in the exhausted group (EG), and those who answered "Yes" were placed in the nonexhausted group (non-EG). The SOF index has been found to be as reliable and valid as the Cardiovascular Health Study (CHS) index which is used most in the world, and this is reliability. In fact, Ensrud et al. showed that in the SOF index predicted disability, fracture, and death as well as the more complex CHS index even though the SOF index is a simple index ${ }^{5,20)}$.

To measure gait speed, participants were asked to walk on a straight walkway (flat floor, $11 \mathrm{~m}$ long) at a comfortable and consistent gait speed. Gait speed was measured over a $5 \mathrm{~m}$ distance from the midpoint of the walkway. The capacity for gait endurance was assessed using the 6 min walk test (6MWT), which was performed as described previously ${ }^{21)}$. Participants were instructed to walk as far as possible in $6 \mathrm{~min}$ along a $10 \mathrm{~m}$ course. The distance (in meters) walked during the 6 min was recorded as the gait endurance. Licensed and well-trained physical therapists assessed these physical performance tests.

The life space of older adults was assessed using a Japanese translation of Life-Space Assessment (LSA) ${ }^{22}, 23$. Scores on the LSA range from 0 to 120 , with higher scores reflecting a larger life space. The frequency of movement (how many days per week) for the five different life space levels was reported by the participants for four weeks before the assessment. These levels are (1) rooms of the home besides where one sleeps; (2) an area directly outside the home (e.g., porch); (3) places in one's neighborhood, other than one's yard or apartment building; (4) places outside one's neighborhood but within one's town; and (5) places outside one's town. Participants were also asked whether they had assistance from another person or devices to reach each level of life space. The overall life space score was computed by summing the products of the each life space level (1-5) multiplied by: (a) degree of independence $(2=$ independent, $1.5=$ used equipment, $1=$ had personal assistance) and by (b) frequency of attainment ( $1=$ less than once per week, $2=1-3$ times per week, $3=4-6$ times per week, 4 = daily).

Differences between groups were examined using the Wilcoxon signed-rank test for nonparametric data or Student's t test for parametric data. Only sex (dichotomous outcome) was analyzed using a $\chi^{2}$ test. Then, multivariate logistic regression analysis was used to investigate whether the potential determinants were associated independently with self-reported exhaustion, and odds ratios (OR) and $95 \%$ confidence intervals $(95 \% \mathrm{CI})$ were calculated. In the adjusted model, we examined the association of each variable (gait speed, gait endurance, and life space) with self-reported exhaustion status while accounting for all covariates (sex, age, BMI, number of medications, and MMSE score). In the fully adjusted model, we then identified which variables were independently related to self-reported exhaustion status when adjusted for gait speed, 6MWT result, LSA score, and all covariates. All analyses were performed using the IBM SPSS (Version 20; IBM Corp., Chicago, IL, USA). Significance was accepted at $\mathrm{p}<0.05$. 
Table 1. Characteristics of participants in EG and non-EG

\begin{tabular}{lcccc}
\hline & $\begin{array}{c}\text { All participants } \\
(\mathrm{n}=356)\end{array}$ & $\begin{array}{c}\text { non-EG } \\
(\mathrm{n}=294)\end{array}$ & $\begin{array}{c}\text { EG } \\
(\mathrm{n}=62)\end{array}$ & p value \\
\hline Sex (number of female) [n (\%)] & $181(50.8)$ & $145(49.3)$ & $36(58.1)$ & $0.26^{\mathrm{a}}$ \\
Age (years) [mean $\pm \mathrm{SE}]$ & $71.6 \pm 0.3$ & $71.5 \pm 0.3$ & $71.9 \pm 0.7$ & 0.61 \\
BMI (kg/m2) [mean $\pm \mathrm{SE}]$ & $23.4 \pm 0.2$ & $23.4 \pm 0.2$ & $23.4 \pm 0.2$ & 0.80 \\
Medications (number of medication) [mean $\pm \mathrm{SE}]$ & $2.13 \pm 0.11$ & $2.05 \pm 0.11$ & $2.50 \pm 0.29$ & 0.11 \\
MMSE (score) [mean $\pm \mathrm{SE}]$ & $26.7 \pm 0.1$ & $26.7 \pm 0.1$ & $27.1 \pm 0.2$ & 0.10 \\
LSA (score) & $90.4 \pm 0.8$ & $91.8 \pm 0.9$ & $83.9 \pm 2.2$ & $<0.01$ \\
6MWT (m) & $449.5 \pm 4.1$ & $453.2 \pm 4.6$ & $432.1 \pm 9.1$ & $<0.05$ \\
Normal gait speed (m/sec) & $1.36 \pm 0.01$ & $1.37 \pm 0.01$ & $1.33 \pm 0.03$ & 0.16 \\
\hline
\end{tabular}

EG, Exhaustion group; SE, standard error; BMI, body mass index; MMSE, Mini-Mental State Examination; LSA, Life Space Assessment; 6MWT, 6 minutes walking test. a was $\chi^{2}$ test and the other variables were student $t$ test. Significance set at $\mathrm{p}<0.05$.

Table 2. Univariate and multiple logistic regression analyses showing the cross-sectional associations between exhaustion (dependent variable) and life-space assessment (independent variable) adjusted for the participants' characteristics

\begin{tabular}{lcccccc}
\hline & \multicolumn{3}{c}{ Adjusted model $^{\mathrm{a}}$} & \multicolumn{3}{c}{ Fully adjusted model $^{\mathrm{b}}$} \\
\cline { 2 - 7 } & OR & $95 \%$ CI & p-value & OR & $95 \%$ CI & p-value \\
\hline LSA (score) & 0.97 & $0.95-0.99$ & $<0.01$ & 0.97 & $0.95-0.99$ & $<0.01$ \\
6MWT (m) & 0.99 & $0.99-0.99$ & 0.04 & 0.99 & $0.99-1.00$ & 0.31 \\
Normal gait speed (m/sec) & 0.33 & $0.08-1.45$ & 0.14 & 0.74 & $0.12-4.68$ & 0.75 \\
\hline
\end{tabular}

All analyses were adjusted for age, gender, BMI, number of medications, and Mini-Mental State Examination score.

OR: odds ratio, CI: confidence interval, LSA: Life-Space Assessment, 6MWT: 6 min walking test.

${ }^{a}$ :Each variablewasincluded as anindependent variable. ${ }^{\mathrm{b}}$ :All variables wereincluding using a forced-entrymethod.

Significance set at $\mathrm{p}<0.05$

\section{RESULTS}

The characteristics of the participants in the non-EG and EG are summarized in Table 1. Sixty-two participants were classified into the EG, giving a $17.4 \%$ prevalence of self-reported exhaustion. The participants' characteristics, including the MMSE score, did not differ significantly between the groups. The LSA scores and the 6MWT results (but not normal gait speed) were significantly lower ( $\mathrm{p}<$ $0.01, p<0.05$, respectively) in the EG compared with the non-EG (Table 1).

The results of the logistic regression analyses are shown in Table 2. In the adjusted model, the LSA score $(\mathrm{OR}=0.97$, 95\% CI; 0.95-0.99) and 6MWT results $(\mathrm{OR}=0.99,95 \% \mathrm{CI}$; 0.99-0.99) were independently associated with exhaustion status. However, in the fully adjusted model (model 3), only the LSA score was independently associated with exhaustion status $(\mathrm{OR}=0.97,95 \% \mathrm{CI} ; 0.95-0.99)$.

\section{DISCUSSION}

The results of this study showed that the prevalence of self-reported exhaustion was $17.4 \%$. A previous study reported that the prevalence of self-reported exhaustion was $14.3 \%$ among older males and $20.4 \%$ among older females ${ }^{15)}$. The prevalence among older adults with MCI in our study was similar to that of healthy older adults. People with MCI who self-reported exhaustion exhibited lower gait endurance performance and a lower LSA score compared with those without exhaustion. In the logistic analyses adjusted for covariates, the LSA score was significantly negatively associated with self-reported exhaustion, although there was no significant relationship between exhaustion and gait speed or gait endurance.

LSA comprises both the frequency and range of outdoor activities. With respect in the frequency of going outdoors, frequency going outdoors was positively associated with health status in older adults including psychological health, such as self-reported exhaustion, compared with older people who were housebound. Going outdoors frequently is positively associated with psychological health but not with physical function or ability to perform $\mathrm{ADL}^{24,25)}$. On the other hand, infrequently going outdoors is negatively associated with health problems among older people 26,27$)$. Going outdoors less than once a week is linked to poorer psychological status, physical function, and ADL ability compared with going outdoors 2-3 times per week ${ }^{24)}$. In the present study, the LSA score was lower in the EG compared with the non-EG. Our current findings complement those of previous studies because we found an association between exhaustion status and LSA score even after adjusting for physical function and other covariates.

In the fully adjusted multivariate regression analysis, self-reported exhaustion was independently associated with decreased life space but not with gait speed or gait endurance. Considering the effect of exhaustion on the capacity 
of energy in the physical activity cycle, older adults cease to perform activities that are not necessary for daily life to prevent exhaustion ${ }^{9}$. In the present study, expanding the life space may not be as important as maintaining gait function for ADL; thus, older adults with self-reported exhaustion would have incorporated a smaller life space. On the other hand, maintaining gait speed or gait endurance may be considered to be more necessary for daily living for older adults than expanding life space and thus were maintained despite self-reported exhaustion.

In the present study, self-reported exhaustion was not associated with physical function including normal gait speed or the results of the 6WMT. The discrepancy between our results and those of other studies may reflect differences in methodology, although previous studies showed an association between self-reported exhaustion and physical function. Other studies have assessed gait speed using maximum speed ${ }^{10)}$ and gait endurance using the $400 \mathrm{~m}$ walking test $^{11)}$, whereas our study included a comfortable speed test and the 6MWT, respectively. Another explanation for the discrepancies may relate to the differences in the populations' characteristics. For example, previous studies have sampled older adults between 65 and 102 years of age (mean 75 years $)^{10)}$ or have targeted only persons of 75 years of age ${ }^{11)}$, whereas the mean age was 71.7 years in our study. The influence of these variables should be investigated using a similar methodology in future studies.

To our knowledge, our study is the first to report on the association between exhaustion status and life space among older adults with MCI. LSA was developed to evaluate mobility status by measuring the life space for elderly individuals living in a community ${ }^{22}$. However, the LSA score is influenced by physical activity as well as physical performance, $\mathrm{ADL}$, and sociodemographic factors ${ }^{28}$. For example, the life space is sensitive to marginal limitations before an older person experiences difficulties in performing $\mathrm{ADL}$ or instrumental $\mathrm{ADL}^{22}$. Life space may be a useful identifier of older adults at risk for cognitive decline ${ }^{29}$. In a previous study, life space was related more closely to cognitive function than to physical performance among older adults with amnestic $\mathrm{MCI}^{30}$. Self-reported exhaustion has also been reported to be associated with cognitive function $^{31)}$. We found that self-reported exhaustion was negatively related to life space among older adults with MCI. Because both a small life space and self-reported exhaustion adversely affect cognitive function, the combination of a small life space and exhaustion may indicate a high risk for cognitive decline. Thus, it may be important to assess not only cognitive function but also self-reported exhaustion for older adults with MCI.

Several limitations in this study should be mentioned. First, the analysis was based on a cross-sectional design, and we were therefore able to examine only the relationships with exhaustion. Second, we did not collect data on other factors that may influence one's feeling of exhaustion such as the cortisol level, which is a biomarker for the "history" of stressful life events ${ }^{32}$. This factor and others should be examined in future studies of exhaustion in older people.
In summary, older adults with MCI who have a restricted life space had self-reported exhaustion. Less life space may lead to several health problems such as disability or dementia in older adults with MCI. Evaluation and preventive strategies to target self-reported exhaustion are recommended to help maintain health status in this population. Future research is needed to identify ways for older people with MCI to improve their exhaustion status.

\section{REFERENCES}

1) Petersen RC: Mild cognitive impairment as a diagnostic entity. J Intern Med, 2004, 256: 183-194. [Medline] [CrossRef]

2) Petersen RC, Doody R, Kurz A, et al.: Current concepts in mild cognitive impairment. Arch Neurol, 2001, 58: 1985-1992. [Medline] [CrossRef]

3) Palmer K, Wang HX, Bäckman L, et al.: Differential evolution of cognitive impairment in nondemented older persons: results from the Kungsholmen Project. Am J Psychiatry, 2002, 159: 436-442. [Medline] [CrossRef]

4) Boyle PA, Buchman AS, Wilson RS, et al.: Physical frailty is associated with incident mild cognitive impairment in community-based older persons. J Am Geriatr Soc, 2010, 58: 248-255. [Medline] [CrossRef]

5) Ensrud KE, Ewing SK, Taylor BC, et al.: Comparison of 2 frailty indexes for prediction of falls, disability, fractures, and death in older women. Arch Intern Med, 2008, 168: 382-389. [Medline] [CrossRef]

6) Fried LP, Tangen CM, Walston J, et al. Cardiovascular Health Study Collaborative Research Group: Frailty in older adults: evidence for a phenotype. J Gerontol A Biol Sci Med Sci, 2001, 56: M146-M156. [Medline] [CrossRef]

7) Raji MA, Al Snih S, Ostir GV, et al.: Cognitive status and future risk of frailty in older Mexican Americans. J Gerontol A Biol Sci Med Sci, 2010, 65: 1228-1234. [Medline] [CrossRef]

8) Xue QL, Bandeen-Roche K, Varadhan R, et al.: Initial manifestations of frailty criteria and the development of frailty phenotype in the Women's Health and Aging Study II. J Gerontol A Biol Sci Med Sci, 2008, 63: 984 990. [Medline] [CrossRef]

9) Schrack JA, Simonsick EM, Ferrucci L: The energetic pathway to mobility loss: an emerging new framework for longitudinal studies on aging. J Am Geriatr Soc, 2010, 58: S329-S336. [Medline] [CrossRef]

10) Mänty M, Ekmann A, Thinggaard M, et al.: Fatigability in basic indoor mobility in nonagenarians. J Am Geriatr Soc, 2012, 60: 1279-1285. [Medline] [CrossRef]

11) Vestergaard S, Nayfield SG, Patel KV, et al.: Fatigue in a representative population of older persons and its association with functional impairment, functional limitation, and disability. J Gerontol A Biol Sci Med Sci, 2009, 64: 76-82. [Medline] [CrossRef]

12) Newton JL, Pairman J, Hallsworth K, et al.: Physical activity intensity but not sedentary activity is reduced in chronic fatigue syndrome and is associated with autonomic regulation. QJM, 2011, 104: 681-687. [Medline] [CrossRef]

13) Constant EL, Adam S, Gillain B, et al.: Cognitive deficits in patients with chronic fatigue syndrome compared to those with major depressive disorder and healthy controls. Clin Neurol Neurosurg, 2011, 113: 295-302. [Medline] [CrossRef]

14) Laurin D, Verreault R, Lindsay J, et al.: Physical activity and risk of cognitive impairment and dementia in elderly persons. Arch Neurol, 2001, 58: 498-504. [Medline] [CrossRef]

15) Chen MK: The epidemiology of self-perceived fatigue among adults. Prev Med, 1986, 15: 74-81. [Medline] [CrossRef]

16) Shimada H, Makizako H, Doi T, et al.: Combined prevalence of frailty and mild cognitive impairment in a population of elderly Japanese people. J Am Med Dir Assoc, 2013, 14: 518-524. [Medline] [CrossRef]

17) Folstein MF, Folstein SE, McHugh PR: "Mini-mental state". A practical method for grading the cognitive state of patients for the clinician. J Psychiatr Res, 1975, 12: 189-198. [Medline] [CrossRef]

18) Makizako H, Shimada H, Park H, et al.: Evaluation of multidimensional neurocognitive function using a tablet personal computer: test-retest reliability and validity in community-dwelling older adults. Geriatr Gerontol Int, 2013, 13: 860-866. [Medline] [CrossRef]

19) Sheikh JI, Yesavage JA: Geriatric Depression Scale (GDS) recent evidence and development of a shorter violence. Clin Gerontol, 1986, 5: 165-173. [CrossRef]

20) Ensrud KE, Ewing SK, Cawthon PM, et al. Osteoporotic Fractures in Men Research Group: A comparison of frailty indexes for the prediction of falls, 
disability, fractures, and mortality in older men. J Am Geriatr Soc, 2009, 57: 492-498. [Medline] [CrossRef]

21) Crapo RO, Casaburi R, Coates AL, et al. ATS Committee on Proficiency Standards for Clinical Pulmonary Function Laboratories: ATS statement: guidelines for the six-minute walk test. Am J Respir Crit Care Med, 2002, 166: 111-117. [Medline] [CrossRef]

22) Baker PS, Bodner EV, Allman RM: Measuring life-space mobility in community-dwelling older adults. J Am Geriatr Soc, 2003, 51: 1610-1614. [Medline] [CrossRef]

23) Harada K, Shimada H, Sawyer P, et al.: [Life-space of community-dwelling older adults using preventive health care services in Japan and the validity of composite scoring methods for assessment]. Nippon Koshu Eisei Zasshi, 2010, 57: 526-537. [Medline]

24) Fujita K, Fujiwara Y, Kumagai S, et al.: [The frequency of going outdoors, and physical, psychological and social functioning among communitydwelling older adults]. Nippon Koshu Eisei Zasshi, 2004, 51: 168-180. [Medline]

25) Simonsick EM, Guralnik JM, Volpato S, et al.: Just get out the door! Importance of walking outside the home for maintaining mobility: finding from the women's health and aging study. J Am Geriatr Soc, 2005, 53: 198-203. [Medline] [CrossRef]

26) Ganguli M, Fox A, Gilby J, et al.: Characteristics of rural homebound older adults: a community-based study. J Am Geriatr Soc, 1996, 44: 363-370.
[Medline]

27) Doi $T$, Ono R, Ono K, et al.: The association between fear of falling and physical activity in older women. J Phys Ther Sci, 2012, 24: 859-862. [CrossRef]

28) Peel C, Sawyer Baker P, Roth DL, et al.: Assessing mobility in older adults: the UAB study of aging life-space assessment. Phys Ther, 2005, 85: 10081119. [Medline]

29) Crowe M, Andel R, Wadley VG, et al.: Life-space and cognitive decline in a community-based sample of African American and Caucasian older adults. J Gerontol A Biol Sci Med Sci, 2008, 63: 1241-1245. [Medline] [CrossRef]

30) Uemura K, Shimada H, Makizako H, et al.: Factors associated with lifespace in older adults with amnestic mild cognitive impairment. Geriatr Gerontol Int, 2013, 13: 161-166. [Medline] [CrossRef]

31) Morgan CA 3rd, Russell B, McNeil J, et al.: Baseline burnout symptoms predict visuospatial executive function during survival school training in special operations military personnel. J Int Neuropsychol Soc, 2011, 17: 494-501 [CrossRef]. [Medline]

32) Glise K, Hadzibajramovic E, Jonsdottir IH, et al.: Self-reported exhaustion: a possible indicator of reduced work ability and increased risk of sickness absence among human service workers. Int Arch Occup Environ Health, 2010, 83: 511-520. [Medline] [CrossRef] 